\title{
Design and Control of Optimal Feedforward Trajectories for Scanners: STM Example. ${ }^{1}$
}

\author{
$\begin{array}{lll}\text { H. Perez } & & \text { Q. Zou } \\ & & \text { S. Devasia }\end{array}$
}

\begin{abstract}
This article addresses the design and control of optimal scan trajectories, which is important in applications such as the imaging of nano-scale surface phenomena using Scanning Tunneling Microscopes (STM), quick-return mechanisms and cams used in manufacturing, and general repeating processes. In this article we pose the problem of designing the output trajectory for a scanner such that the input energy is minimized. The problem is solved by first integrating optimal control techniques with a model-based inversion approach. After this integration, the input-energy for the entire scan is then minimized to solve the optimal scanning problem. Additionally, the method is applied to highspeed scanning of an STM, which is a key tool in emerging nanotechnologies. Simulation and experimental results are presented to illustrate the technique and its advantages.
\end{abstract}

\section{Introduction}

A typical scanning operation consists of two sections: (1) a first section during which a desired output trajectory is tracked precisely - this is often referred to as the activescan section or the tracking section; and (2) a second section which aims to achieve a quick return of the output to a predefined value so that the active scan can be repeated - this is the retrace section or the output-transition section. These tracking and transition sections are repeated in a scanning operation; such scanning is used in a variety of applications, for example, in the imaging of nano-scale surface phenomena using Scanning Tunneling Microscopes (STM) [1], in quick-return mechanisms and cams used in manufacturing [2], in repeating processes such as paper-feed operation in copiers [3], and in the control of flexible structures such as long robotic arms $[4,5]$. While precision control of the tracking section can be achieved using model-based inversion techniques, the design of the output trajectory during the transition section is often ad hoc, and is usually accomplished using smoothing techniques and polynomial-based techniques $[6,5,3]$. In this article we pose the problem of designing the output trajectory for a scanner such that the total energy (i.e. during tracking and transition) is minimized. The method is applied to high-speed scanning of a Scanning Tunneling Microscope (STM), which is a key tool in emerging nanotechnologies to investigate and control nano-scale surface phenomena $[7,8]$. Experimental and

\footnotetext{
${ }^{1}$ Support from NASA Ames Research Center Grant NAG 21450 and NSF Grant CMS 0196214 is acknowledged

2Email: hrpr@u.washington.edu. Mechanical Engineering Department. Univ. of Washington

${ }^{3}$ Email: qzouatuw@u.washington.edu. Mechanical Engineering Department. Univ. of Washington

4 Email: devasia@u.washington.edu. Mechanical Engineering Department. Univ. of Washington Fax: 2066858047
}

simulation results are presented to illustrate the technique and its advantages.

If the desired scanning trajectory is known, then several approaches are available for finding inputs that track the desired output trajectory; these methods include repetitive and iterative control techniques, e.g., see $[9,10,11]$. However, in many scanning operations, the output trajectory during the output-transition section is unknown - the trajectory design problem is part of the controller design problem. Optimal transitions have been studied in control theory [12] when the initial and final states are pre-specified; however, in a scanning problem the boundary states at the initiation and completion of the output-transition are not specified (only the output at these instants are known and not the states). This problem of unknown boundary states can be circumvented by choosing a smooth output trajectory during the output transition section. For example, spectral windows proposed in [6] can be used to obtain a smooth output-trajectory during the output-transition section. Another approach is to design the output trajectory using polynomial functions for smooth transitions between tracking and transition sections [5]. However, these methods are ad-hoc, and are not optimal. Thus, a systematic approach to output-trajectory design and control for scanners is not available at present.

The contribution of this article is a systematic solution of the optimal output-trajectory design and control problem for scanners. The unknown boundary states, i.e., initial and final states for the output transition section (retraces section), are considered as variables to be optimized in this article. It is show that portions of the boundary states (for the retrace section) can be determined from the tracking requirements of the active-scan section. The remaining portions of the boundary states are then optimized to minimize the overall input energy required for scanning. Thus, the proposed approach integrates standard optimal control approaches with the model-based inversion approach to solve the optimal scanning problem. While the article focuses on minimizing the input energy (which is a standard optimalcontrol cost criterion), the proposed integration of inversion and optimization approaches to optimally design the scan trajectory is general; the proposed methodology can be used to minimize other criterion such as (a) the time for the output transition and (b) motion-induced vibrations in the system during the transition.

The article is organized in the following format. In Section 2 , we formulate the optimal scanning problem. Section 3 solves the optimal scanning problem by integrating a modelbased inversion approach with the state-to-state transition 
approach. Section 4 describes the Scanning Tunneling Microscope (STM) used in the experiments, and describes the application of the optimal scanning approach to the STM. Section 5 presents simulation and experimental results and discussions. Conclusions are in Section 6.

\section{Problem formulation}

Consider a linear, time-invariant system described by

$$
\begin{aligned}
& \dot{x}(t)=A x(t)+B u(t) \\
& y(t)=C x(t),
\end{aligned}
$$

where $x(t) \in \Re^{n}$ is the state, $u(t) \in \Re^{p}$ is the input and $y(t) \in \Re^{p}$ is the output. Note that the system is square with the same number of inputs as outputs. In the following, it is assumed that (1) the system (Eq. 1) has a well defined relative degree $\rho:=\left[\rho_{1}, \rho_{2}, \ldots, \rho_{p}\right]$ (e.g., see reference [13]), and (2) system (Eq. 1) is controllable. The scanning problem is stated, next.

Definition 1 The Scanning Problem Given (1) a desired output trajectory $y_{\text {track }}(t)$ to be tracked during the tracking section $t_{0}<t<t_{i}$ and (2) the output-transition time interval $\left[t_{i}, t_{f}\right]$, find a bounded input-state trajectory $\left[u_{f f}(\cdot), x_{r e f}(\cdot)\right]$ such that the following three requirements are met.

1. The system equations are satisfied during scanning

$$
\dot{x}_{r e f}(t)=A x_{r e f}(t)+B u_{f f}(t), \quad t_{0} \leq t \leq t_{f}
$$

2. Exact output tracking is achieved during the tracking (active-scan) section, i.e.,

$$
y_{\text {track }}(t)=C x_{r e f}(t), \quad t_{0}<t<t_{i}
$$

3. During the transition section, $t_{i} \leq t \leq t_{f}$, an output transition is performed to reset the system's output before the start of the next scan cycle. Therefore, the output values at the start and end of the output transition are

$$
\begin{aligned}
y_{\text {tran }}\left(t_{i}\right) & :=y\left(t_{i}\right)=C x_{\text {ref }}\left(t_{i}\right) \\
& =\lim _{t \rightarrow t_{i}^{-}} y_{\text {track }}(t):=\bar{y} \\
y_{\text {tran }}\left(t_{f}\right) & :=y\left(t_{f}\right)=C x_{\text {ref }}\left(t_{f}\right) \\
& =\lim _{t \rightarrow t_{0}^{+}} y_{\text {track }}(t):=\underline{y}
\end{aligned}
$$

Definition 2 Optimal Scanning Problem

Find a bounded input-state trajectory $\left[u_{f f}(\cdot), x_{r e f}(\cdot)\right]$ that solves the Scanning Problem (see Definition 1), and minimizes the following cost function over possible inputs

$$
\begin{aligned}
& J_{\text {scanning }}\left(t_{0}, t_{i}, t_{f}, u\right):=\int_{t_{0}}^{t_{f}} u^{T} R u d t \\
&=\int_{t_{0}}^{t_{i}} u_{\text {track }}^{T} R u_{\text {track }} d t+ \\
& \int_{t_{i}}^{t_{f}} u_{\text {tran }}^{T} R u_{\text {tran }} d t \\
&:= J_{\text {track }}+J_{\text {tran }}
\end{aligned}
$$

where $u_{\text {track }}$ is the input during the tracking section $\left(t_{0}, t_{i}\right), u_{\text {tran }}$ is the input during the transition section $\left[t_{i}, t_{f}\right], R$ is a positive-definite, real, symmetric matrix, and

$$
\begin{aligned}
J_{\text {track }} & :=\int_{t_{0}}^{t_{i}} u_{\text {track }}^{T} R u_{\text {track }} d t ; \\
J_{\text {track }} & :=\int_{t_{0}}^{t_{i}} u_{\text {track }}^{T} R u_{\text {track }} d t ;
\end{aligned}
$$

\section{Solution to the Optimal Scanning Problem}

We solve the optimal scanning problem in three steps: (Step 1) parameterize the exact-tracking input $u_{\text {track }}$ and the associated cost $J_{\text {track }}$ during the tracking section in terms of the boundary states $\left(x\left(t_{i}\right), x\left(t_{f}\right)\right)$; (Step 2) parameterize the optimal cost, $J_{\text {tran }}$, and the input $u_{\text {tran }}$ during the transition section in terms of the boundary states $\left(x\left(t_{i}\right), x\left(t_{f}\right)\right)$; and (Step 3) optimally choose the boundary states $\left(x\left(t_{i}\right), x\left(t_{f}\right)\right)$ to solve the optimal scanning problem, i.e., find the minimal $J_{\text {scanning }}$.

3.1 Step 1. Cost during the tracking section The exact tracking input for a given output trajectory can be obtained by using model-based inversion approaches. We begin by rewriting the system equations in the outputtracking form (see, e.g., [13] Chapter 9, or [14]).

3.1.1 Output Tracking Form: If the system has a well-defined relative degree assumption, there exists

1. A coordinate transformation, $\Phi$

$$
\Phi\left[\begin{array}{c}
\xi \\
\eta_{s} \\
\eta_{u}
\end{array}\right]=x
$$

2. and an input law $u(t)=u_{f f}(t), t_{0}<t<t_{i}$

$$
u_{f f}(t)=\hat{B}_{s} \eta_{s}(t)+\hat{B}_{u} \eta_{u}(t)+\hat{B}_{\xi} Y_{d}(t)
$$

that transforms the system (Eq. 1) into the output-tracking form (see, e.g., $[13,14]$ )

$$
\begin{aligned}
\dot{\xi}(t) & =\dot{\xi}_{d}(t) \\
{\left[\begin{array}{c}
\dot{\eta}_{s} \\
\dot{\eta}_{u}
\end{array}\right] } & =\left[\begin{array}{cc}
A_{s} & 0 \\
0 & A_{u}
\end{array}\right]\left[\begin{array}{l}
\eta_{s} \\
\eta_{u}
\end{array}\right]+\left[\begin{array}{c}
B_{s} \\
B_{u}
\end{array}\right] Y \\
y & =\hat{C} \xi
\end{aligned}
$$

where

1. the output and its time-derivatives upto order $\rho-1:=$ $\left[\rho_{1}-1, \rho_{2}-1, \ldots, \rho_{p}-1\right]$ are denoted by

$$
\begin{aligned}
\xi:= & {\left[y_{1}, \dot{y}_{1}, \ldots, \frac{d^{\rho_{1}-1} y_{1}}{d t^{\rho_{1}-1}}, y_{2}, \dot{y}_{2}, \ldots, \frac{d^{\rho_{2}-1} y_{2}}{d t^{\rho_{2}-1}}\right.} \\
& \left., \ldots, y_{p}, \dot{y}_{p}, \ldots, \frac{d^{\rho_{p}-1} y_{p}}{d t^{\rho_{p}}-1}\right]^{T}
\end{aligned}
$$

Furthermore, when a sufficiently smooth desired output trajectory is specified

$$
\xi_{d}(t):=\left.\xi(t)\right|_{y(\cdot)=y_{d}(\cdot)} .
$$


2. The output and its time-derivatives upto order $\rho:=$ $\left[\rho_{1}, \rho_{2}, \ldots, \rho_{p}\right]$ are denoted by

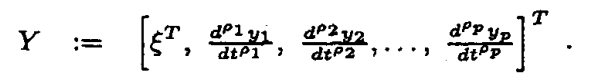

Again, if the desired output is specified then

$$
Y_{d}(t):=\left.Y\right|_{y(\cdot)=y_{d}(\cdot)}
$$

3. The internal dynamics is represented by $\eta_{s}$ and $\eta_{u}$. The internal dynamics is assumed to be decoupled (without loss of generality); the stable subspace of the internal dynamics is $\eta_{s}$ and the remaining dynamics is represented by $\eta_{\psi}$. The corresponding submatrix $A_{s}$ has its eigenvalues in the open left half of the complex plane, and the submatrix $A_{u}$ has its eigenvalues in the right half of the complex plane which includes the imaginary axis.

Remark 1 The desired output trajectory has to be differentiable a certain number of times depending on the relative degree of the system. Such differentiability of the desired output trajectory is necessary for invertibility (i.e., exact output tracking); however, the well-defined relative degree may be replaced by general invertibility conditions with appropriate changes in the procedures to obtain the inverse system and the internal dynamics (see, e.g., $(15,16)$ ).

3.1.2 Input $u_{\text {track }}$ during the tracking section $t_{0}<t<t_{i}$ : The input control law (Eq. 6) for exact tracking is completely specified in terms of the internal dynamics $\left(\eta_{s}, \eta_{u}\right)$ and the desired trajectory. Therefore, for a given desired output trajectory, finding the exact tracking input is equivalent to finding bounded solutions to the internal dynamics. However, finding a bounded solution to the internal dynamics (Eq. 8) can be challenging because $A_{u}$ has eigenvalues in the right half of the complex plane. We use stable inversion approach (i.e., integrate $\eta_{u}$ backward in time) to find bounded solutions to the internal dynamics as $[17,14]$

$$
\begin{gathered}
\eta_{s}(t)=e^{A_{s}\left(t-t_{0}\right)} \eta_{s}\left(t_{0}\right)+\int_{t_{0}}^{t} e^{A_{s}(t-\lambda)} B_{s} Y_{d} d \lambda, \\
t_{0}<t<t_{i}
\end{gathered}
$$

Substituting this solution to the internal dynamics into the control law (Eq. 6) the exact tracking input becomes

$$
\begin{aligned}
u_{\text {track }}= & {\left[\begin{array}{ll}
\hat{B}_{s} e^{A_{s}\left(t-t_{0}\right)} & \hat{B}_{u} e^{-A_{u}\left(t_{i}-t\right)}
\end{array}\right]\left[\begin{array}{c}
\eta_{s}\left(t_{f}\right) \\
\eta_{u}\left(t_{i}\right)
\end{array}\right]+} \\
& \hat{B}_{s} \int_{t_{0}}^{t} e^{A_{g}(t-\lambda)} B_{s} Y_{d}(t) d \lambda- \\
& \hat{B}_{u} \int_{t}^{t_{i}} e^{-A_{u}(\lambda-t)} B_{u} Y_{d}(t) d \lambda+\hat{B}_{\xi} Y_{d}(t) \\
= & P(t) \Psi+S\left(t, Y_{d}(t)\right)
\end{aligned}
$$

where $\Psi$ is defined as the boundary condition

$$
\Psi:=\left[\begin{array}{l}
\eta_{s}\left(t_{0}\right) \\
\eta_{u}\left(t_{i}\right)
\end{array}\right]=\left[\begin{array}{l}
\eta_{s}\left(t_{f}\right) \\
\eta_{u}\left(t_{i}\right)
\end{array}\right] .
$$

3.1.3 Cost $J_{\text {track }}$ during the tracking section, $t_{0}<t<t_{i}:$

Lemma 1 Tracking cost $J_{\text {track }}$ during the tracking section, $t_{0}<t<t_{i}$

The cost $J_{\text {track }}$ during the tracking section is completely specified in terms of the boundary condition $\Psi$ (Eq. 17). Furthermore the cost is quadratic in $\Psi$ and is given by

$$
\begin{aligned}
J_{\text {track }}\left(t_{0}, t_{i}, \Psi\right) & =\int_{t_{0}}^{t_{i}} u_{s c a n}^{T} R u_{s c a n} d t \\
= & \Psi^{T}\left(\int_{t_{0}}^{t_{i}} P(t)^{T} R P(t) d t\right) \Psi- \\
& 2 \Psi^{T}\left(-\int_{t_{0}}^{t_{i}} P(t)^{T} R S\left(t, Y_{d}(t)\right) d t\right)+ \\
& \int_{t_{0}}^{t_{i}} S\left(t, Y_{d}(t)\right)^{T} R S\left(t, Y_{d}(t)\right) d t \\
:= & \Psi^{T} \Lambda_{\text {track }} \Psi-2 \Psi^{T} b_{\text {track }}+c_{\text {track }}
\end{aligned}
$$

Proof This follows by substituting the control law (Eq. 16) into the tracking cost $J_{\text {track }}$ (Eq. 3).

3.2 Step 2. Cost during the transition section, $t_{i} \leq$ $t \leq t_{f}$

We show that the transition cost $J_{\text {tran }}$ is quadratic in the boundary state $\Psi$ which determines the boundary states $\left(x\left(t_{i}\right), x\left(t_{f}\right)\right)$ for the output transition.

Remark 2 The boundary condition $\Psi$ determines the boundary states $\left(x\left(t_{i}\right), x\left(t_{f}\right)\right)$ for the output transition as

$$
x\left(t_{i}\right)=\Phi\left[\begin{array}{lll}
\xi\left(t_{i}\right) & \eta_{s}\left(t_{i}\right) & \eta_{u}\left(t_{i}\right)
\end{array}\right]^{T}
$$

and

$$
x\left(t_{f}\right)=\Phi\left[\begin{array}{lll}
\xi\left(t_{f}\right) & \eta_{s}\left(t_{f}\right) & \eta_{u}\left(t_{f}\right)
\end{array}\right]^{T}
$$

where $\eta_{s}\left(t_{i}\right)$ and $\eta_{u}\left(t_{f}\right)$ are found from $\Psi$ using $E q$. (14) and Eq. (15), respectively as

$$
\begin{aligned}
\eta_{s}\left(t_{i}\right) & =e^{A_{s}\left(t_{i}-t_{0}\right)} \eta_{s}\left(t_{0}\right)+\int_{t_{0}}^{t_{i}} e^{A_{s}\left(t_{i}-\lambda\right)} B_{s} Y d \lambda \\
& :=K_{s} \eta_{s}\left(t_{0}\right)+\Lambda_{s} \\
\eta_{u}\left(t_{f}\right) & =e^{-A_{u}\left(t_{i}-t_{0}\right)} \eta_{u}\left(t_{i}\right)-\int_{t_{0}}^{t_{i}} e^{-A_{u}\left(\lambda-t_{0}\right)} B_{u} Y d \lambda \\
& :=K_{u} \eta_{u}\left(t_{i}\right)+\Lambda_{u}=\eta_{u}\left(t_{0}\right)
\end{aligned}
$$

Lemma 2 Minimal-energy Transition

Given the boundary states $\left(x\left(t_{i}\right), x\left(t_{f}\right)\right)$, the input $u_{\text {tran }}(\cdot)$ that minimizes the cost function $J_{\text {tran }}(E q .4)$ is given by 


$$
\begin{aligned}
& u_{\text {tran }}(t)= \\
& R^{-1} B^{T} e^{A^{T}\left(t_{f}-t\right)} G_{\left(t_{i}, t_{f}\right)}^{-1}\left[x\left(t_{f}\right)-e^{A\left(t_{f}-t_{i}\right)} x\left(t_{i}\right)\right]
\end{aligned}
$$

where $G_{\left(t_{i}, t_{f}\right)}$ is the invertible controllability Gramian given by

$$
G_{\left(t_{i}, t_{f}\right)}=\int_{t_{i}}^{t_{f}} e^{A\left(t_{f}-\tau\right)} B R^{-1} B^{T} e^{A^{T}\left(t_{f}-\tau\right)} d \tau
$$

The minimum cost of transition for given boundary states $\left(x\left(t_{i}\right), x\left(t_{f}\right)\right)$ is given by

$$
\begin{aligned}
& J_{\text {tran }}\left(t_{i}, t_{f}, x\left(t_{i}\right), x\left(t_{f}\right)\right)= \\
& -\left[x\left(t_{f}\right)-e^{A\left(t_{f}-t_{i}\right)} x\left(t_{i}\right)\right]^{T} G_{\left(t_{i}, t_{f}\right)}^{-1}\left[x\left(t_{f}\right)-e^{A\left(t_{f}-t_{i}\right)} x\left(t_{i}\right)\right]
\end{aligned}
$$

Proof See, for example, [12] page 165.

The minimum cost (Eq. 23) and the input (Eq. 21) during the transition depend on the final state difference $d\left(t_{i}, t_{f}\right)=$ $x\left(t_{f}\right)-e^{A\left(t_{f}-t_{i}\right)} x\left(t_{i}\right)$ which can be re-written in terms of the boundary condition $\Psi$ as

$$
\begin{aligned}
d\left(t_{i}, t_{f}\right) & =x\left(t_{f}\right)-e^{A\left(t_{f}-t_{i}\right)} x\left(t_{i}\right) \\
& =\Phi\left[\begin{array}{c}
\xi\left(t_{f}\right) \\
\eta_{s}\left(t_{f}\right) \\
\eta_{u}\left(t_{f}\right)
\end{array}\right]-e^{A\left(t_{f}-t_{i}\right)} \Phi\left[\begin{array}{c}
\xi\left(t_{i}\right) \\
\eta_{s}\left(t_{i}\right) \\
\eta_{u}\left(t_{i}\right)
\end{array}\right] \\
& =\left[\Phi_{\xi}\left|\Phi_{\eta_{s}}\right| \Phi_{\eta_{u}}\right]\left[\begin{array}{c}
\xi\left(t_{f}\right) \\
\eta_{s}\left(t_{f}\right) \\
K_{u} \eta_{u}\left(t_{i}\right)+\Lambda_{u}
\end{array}\right]- \\
& {\left[W_{\xi}\left|W_{\eta_{s}}\right| W_{\eta_{u}}\right]\left[\begin{array}{c}
\xi\left(t_{i}\right) \\
K_{s} \eta_{s}\left(t_{f}\right)+\Lambda_{s} \\
\eta_{u}\left(t_{i}\right)
\end{array}\right] } \\
:= & H_{1} f+H_{2} \Psi
\end{aligned}
$$

Substituting for the final state difference $d\left(t_{i}, t_{f}\right)$ rewritten in terms of the boundary condition $\Psi$ (Eq. 24) into the cost Eq.(23) we obtain the following lemma.

Lemma 3 Transition Cost $J_{\text {tran }}$ during the transition section, $t_{i} \leq t \leq t_{f}$

For a specified boundary condition $\Psi$ (Eq. 17), the transition cost is quadratic in $\Psi$ and is given by

$$
\begin{aligned}
& J_{\text {tran }}\left(t_{i}, t_{f}, \Psi\right)=d\left(t_{i}, t_{f}\right)^{T} G_{\left(t_{i}, t_{f}\right)}^{-1} d\left(t_{i}, t_{f}\right) \\
&=\left(H_{1} f+H_{2} \Psi\right)^{T} G_{\left(t_{i}, t_{f}\right)}^{-1}\left(H_{1} f+H_{2} \Psi\right) \\
&= \Psi^{T} H_{2}^{T} G_{\left(t_{i}, t_{f}\right)}^{-1} H_{2} \Psi+2 \Psi^{T} H_{2}^{T} G_{\left(t_{i}, t_{f}\right)}^{-1} H_{1} f+ \\
& f^{T} H_{1}^{T} G_{\left(t_{i}, t_{f}\right)}^{-1} H_{1} f \\
&:= \Psi^{T} \Lambda_{\text {tran }} \Psi-2 \Psi^{T} b_{t r a n}+c_{t r a n}
\end{aligned}
$$

3.3 Step 3. Solution to the Optimal Scanning Problem

For a given boundary condition $\Psi$ (Eq. 17), the minimum cost for scanning is quadratic in $\Psi$ and can be obtained by adding the tracking cost (Eq. 18) to the minimum transition cost (Eq. 25) as shown below.

$$
\begin{aligned}
J_{\text {scanning }}\left(t_{0}, t_{i}, t_{f}, \Psi\right)= & \Psi^{T}\left(\Lambda_{\text {track }}+\Lambda_{\text {tran }}\right) \Psi- \\
& 2 \Psi^{T}\left(b_{\text {track }}+b_{\text {tran }}\right)+ \\
& \left(c_{\text {track }}+c_{\text {tran }}\right) \\
:= & \Psi^{T} \Lambda \Psi-2 \Psi^{T} b+c
\end{aligned}
$$

Optimal values for boundary condition $\Psi$ are found in the following theorem, which also finds the solution to the optimal scanning problem.

Theorem 1 Optimal solution for the Scanning problem

The solution to the optimal scanning problem can be obtained as follows.

1. The total cost function (Eq. 2) is minimized by the following choice of the boundary condition $\Psi^{*}$

$$
\Psi^{*}=\left[\begin{array}{l}
\eta_{s}\left(t_{0}\right)^{*} \\
\eta_{u}\left(t_{i}\right)^{*}
\end{array}\right]=\Lambda^{\dagger} b
$$

where $\Lambda^{\dagger}$ is the generalized inverse of $\Lambda$ given by

$$
\Lambda^{\dagger}=V\left[\begin{array}{cc}
\Sigma^{-1} & 0 \\
0 & 0
\end{array}\right] U^{*}
$$

where $U, \Sigma$ (invertible) and $V$ defines the singular value decomposition of $\Lambda$ as

$$
\Lambda=U\left[\begin{array}{cc}
\Sigma & 0 \\
0 & 0
\end{array}\right] V^{*}
$$

It is noted that $\Lambda^{\dagger}=0$ if $\Lambda=0$, and that $\Lambda^{\dagger}=\Lambda^{-1}$ if $\Lambda$ is invertible.

2. The optimal input $u_{f f}^{*}$ is given by

$$
\begin{aligned}
u_{f f}^{*}(t)= & u_{t r a c k}^{*} \quad \text { for } t_{0}<t<t_{i} \\
= & {\left[\begin{array}{ll}
\hat{B}_{s} e^{A s\left(t-t_{0}\right)} & \hat{B}_{u} e^{-A_{u}\left(t_{i}-t\right)}
\end{array}\right] \Psi+} \\
& \hat{B}_{s} \int_{t_{0}}^{t} e^{A_{s}(t-\lambda)} B_{s} Y(t) d \lambda- \\
& \hat{B}_{u} \int_{t}^{t_{i}} e^{-A_{u}(\lambda-t)} B_{u} Y(t) d \lambda+\hat{B}_{\xi} Y(t) \\
u_{f f}^{*}(t)= & u_{t r a n}^{*}(t) \quad \text { for } t_{i} \leq t \leq t_{f} \\
= & R^{-1} B^{T^{1}} e^{A^{T}\left(t_{f}-t\right)} G_{\left(t_{i}, t_{f}\right)}^{-1} \\
& {\left[x\left(t_{f}\right)^{*}-e^{A\left(t_{f}-t_{i}\right)} x\left(t_{i}\right)^{*}\right] }
\end{aligned}
$$

3. The corresponding reference state trajectory $x_{\text {ref }}$ is given by

$$
x_{r e f}(t)=\Phi\left[\begin{array}{c}
\xi(t) \\
\eta_{s}(t)^{*} \\
\eta_{u}(t)^{*}
\end{array}\right] \quad \text { if } t_{0}<t<t_{i}
$$




$$
\begin{gathered}
x_{r e f}(t)=e^{A\left(t-t_{i}\right)} x\left(t_{i}\right)^{*}+\int_{t_{i}}^{t}\left\{e^{A(t-\tau)} B u_{t r a n}^{*}(\tau)\right\} d \tau \\
\text { if } t_{i} \leq t \leq t_{f}
\end{gathered}
$$

where

$$
\begin{aligned}
& \eta_{s}(t)^{*}=e^{A_{s}\left(t-t_{0}\right)} \eta_{s}\left(t_{0}\right)^{*}+\int_{t_{0}}^{t} e^{A_{g}(t-\lambda)} B_{s} Y d \lambda \\
& \eta_{u}(t)^{*}=e^{-A_{u}\left(t_{i}-t\right)} \eta_{u}\left(t_{i}\right)^{*}-\int_{t}^{t_{i}} e^{-A_{u}(\lambda-t)} B_{u} Y d \lambda
\end{aligned}
$$

and the boundary states $\left(x\left(t_{i}\right)^{*}, x\left(t_{f}\right)^{*}\right)$ are found using the optimal boundary condition $\Psi^{*}$ as discussed in Remark 2.

Proof. By definition, the cost function $J_{\text {scanning }}$ (Eq. 2) is quadratic, and has a lower bound (zero!). The existence of the lower bound implies that the optimization problem always has at least one solution (e.g., see [18], Theorem 4.2.1 in Chapter 4). The first part of the Theorem follows from optimization of quadratic forms [18]. The second part of the theorem follows from Eq. (16) and Eq. (21). The third part of the Theorem follows from the bounded solution for the internal dynamics given in Eq. (14) and Eq. (15).

\section{Scanning Tunneling Microscope (STM) Application}

The optimal-scanning method is applied to a STM (Metris1000 , Burleigh Instruments, Inc.), which is a key tool in emerging nanotechnologies [7] and is used to investigate nano-scale surface phenomena $[8]$. We begin by describing the STM system and its model, and then present the application of the optimal-scanning method to the STM.

\subsection{Burleigh Metris-1000 STM scanner}

The principle of STM operation is shown in Figure 1. A piezo scanner moves the STM-tip across the sample surface as the tunneling current between the STM-tip and the sample (for an applied voltage) is measured. Therefore, the measured tunneling current is directly related to the sample's surface properties and is used to form STM images of the sample's surface properties. A critical component of a STM system is the piezo scanner that positions the STMprobe over the sample surface (see Figure 1). The piezo scanner can move the STM-probe parallel to the sample surface (X-Y axes) as well as perpendicular to the sample surface (Z-axis). Errors in positioning of the STM-tip can lead to distortions in the acquired images. In particular, movement-induced vibrations can lead to significant positioning errors and image distortions at high-speed operation of the STM. Therefore, precision positioning of the STM-tip is critical

The Scanning Problem The scan pattern used to image the sample surface is shown in Figure 1. During the activescan section (or the tracking section), the $Y$-axis displacement $d_{y}$ is kept constant and the $X$-axis displacement $d_{x}$ is increased from left to right (as shown in Figure 1). During the active scan the tunneling current is measured at a

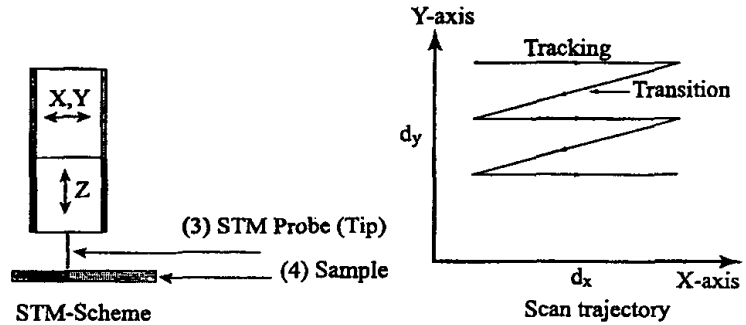

Figure 1: STM scanner Scheme and Scan Trajectory (Figures are not to scale)

constant sampling rate (the STM-tip position is not available for measurement during this scanning process) - the position of the STM-tip needs to be controlled precisely during the active-scan section (e.g., $\mathrm{X}$-axis displacement needs to be tracked exactly) to avoid image-distortions. The active-scan section is followed by the retrace section (transition section) during which the $\mathrm{Y}$-axis displacement is incremented and the $\mathrm{X}$-axis position is returned to the left. The tunneling current information is not measured during the retrace section. This scanning cycle (composed of the tracking and transition sections) is repeated until the desired surface area is imaged. The two sections in the $X$-axis are:

1. The tracking section, $t_{0}<t<t_{i}$, where the tip tracks a desired output trajectory in the $X$-axis. The desired output $y=y_{\text {track }}$ of the system is the displacement $d_{x}$ in the $X$-axis, i.e., $y(t)=d_{x}(t)$. In all the simulations and experiments, the velocity of the desired tracking trajectory was constant while the STM-tip moved in the $X$-axis from $d_{x}=-5 \dot{A}$ (Angstroms) to $d_{x}=5 \dot{A}$.

2. The transition section, $t_{i} \leq t \leq t_{f}$, where the tip performs an output transition in the $X$-axis resetting the tip to the original $X$-axis position. Therefore, the output value for the $X$-axis is taken from the initial value $y_{\operatorname{tran}}\left(t_{i}\right)=y=-5 \dot{A}$ to the final value $y_{\text {tran }}\left(t_{f}\right)=y_{\text {tran }}\left(t_{0}\right)=\bar{y}=5 \dot{A}$.

Remark 3 The scanning movements in the $X$-axis results in movement-induced vibrations, and therefore, it is important to consider the $X$-axis dynamics during the design of the scan trajectories. In contrast, the scanning does not lead to significant distortions in the $Y$ and $Z$ axes positioning. We ignore coupling effects between the $X, Y$, and $Z$ axis if significant dynamic cross-coupling is present then multivariable techniques can be used to model and invert the coupled dynamics. We note that the proposed optimal scanning technique is applicable to the multiple-input multiple-output case. However, we focus this article on the scanning in the $X$-direction

\subsection{STM scanner Modeling}

The dynamics of the STM scanner in the $X$-direction were modeled experimentally using a dynamic signal analyzer 
(DSA) (Standard Research Systems, Model SR785). An input $u$ from the DSA to the STM scanner in the $X$-direction. The STM scanner response was measured using an inductive sensor (Kaman Instruments, Inc., Model SMU 9000). The measured output signal from the inductive sensor was then returned to the DSA. The resulting input-output responses (magnitude and phase responses) at different frequencies were used to construct a model of the STM scanner. To avoid numerical errors, a time-scaling was performed, and the transfer-function model of the STM scanner from the input (applied voltage (volts)) to the displacement in the $\mathrm{X}$-axis $\left(d_{x}[\dot{A}]\right)$ was found as

$$
\frac{d_{x}(s)}{\hat{u}(s)}=\operatorname{Gain} \frac{\prod_{q=1}^{4}\left(s-\text { zeros }_{q}\right)}{\prod_{r=1}^{6}\left(s-\text { poles }_{r}\right)}=G_{S T M}
$$

with zeros, poles and gain given in Table (1).

Table 1: Poles and zeros of the system.

\begin{tabular}{|c|c|c|}
\hline Zeros & Poles & Gain \\
\hline \hline $0.9274 \pm 4.1659 \mathrm{i}$ & $-0.0188 \pm 3.1326 \mathrm{i}$ & 0.1548 \\
$-0.2484 \pm 3.0434 \mathrm{i}$ & $-0.0857 \pm 2.4570 \mathrm{i}$ & \\
& -0.7263 & \\
& -0.3198 & \\
\hline
\end{tabular}

and the Laplace variable $s$ is in $\mathrm{rad} /\left(\mathrm{sec} \times 10^{-4}\right)$. In addition, a first-order low-pass prefilter with $750 \mathrm{~Hz}$ cutoff frequency $\left(G_{f i l t e r}=a /(s+a)\right)$ was used. The pre-filter is used to increase the relative cost of using high frequency inputs (e.g., see frequency weighted cost-functionals developed in [19]). The total transfer function used for designing optimal scan trajectories is given by

$$
\frac{d_{x}(s)}{u(s)}=\frac{0.47124}{s+0.47124} \times G_{S T M}:=G_{\text {system }}
$$

4.3 Solution to the scanning problem

As stated in Definition 1 Page 2, the tracking section (i.e., the active scanning section) has a pre-specified desired trajectory $y_{\text {track}}$; however, the transition section (i.e., the retrace section) doesn't have a pre-specified desired trajectory $y_{\text {tran }}$. There are many ways to perform the transition, therefore, the issue is the choice of a suitable output trajectory for the transition section. Two approaches were used to select the transition trajectory: (1) a polynomial approach and (2) the optimal approach discussed in Section 3.

Approach 1. Polynomial approach to Scanning. The relative degree of the system (Eq. 33) is three, therefore, the state of the system (in the tracking form) contains the output and its first and second time derivatives, $\xi=\left[\begin{array}{lll}y & \frac{d y}{d t} & \frac{d^{2} y}{d t^{2}}\end{array}\right]^{T}$. These three states need to be continuous for a smooth change between the tracking section and transition section at times $t=t_{i}$ and $t=t_{f}$, which yields six boundary conditions on the output trajectory. Therefore, the output trajectory during the transition trajectory is chosen to be a fifth-order polynomial with six unknown co-efficients of the form

$$
y(t)=a_{0}+a_{1} t+a_{2} t^{2}+a_{3} t^{3}+a_{4} t^{4}+a_{5} t^{5}
$$

For this example $a_{0}=5, a_{1}=0.2, a_{2}=0, a_{3}=-0.0016$, $a_{4}=4.8 \times 10^{-5}$, and $a_{5}=3.84 \times 10^{-7}$.

Two methods were applied to track the complete scan trajectory: (1) control that ignores the STM scanner dynamics and (2) control that accounts for the STM scanner dynamics. The results of applying these two approaches is used to demonstrate the need to account for the scanner dynamics in the control design.

1. Control without considering the STM scanner dynamics. In this approach (referred to as the $D C$ gain approach), the input $u$ and output $y$ are related by $y=k u$ where $k=1.9166$ is the low frequency gain (or DC gain) of the STM scanner. The input is chosen as

$$
u_{D C}(t)=\frac{y_{\text {polynomial }}(t)}{k}, \text { for } t_{0} \leq t \leq t_{f}
$$

where $y_{\text {polynomial }}($.$) is the desired output (tracking$ plus transition).

2. Control that accounts for the STM scanner dynamics. The Inversion-based approach was the approach used to account the STM scanner dynamics. The inverse input $u_{i n v}$ that guarantees perfect tracking is

$$
u_{i n v}(t)=G_{s y s t e m}^{-1}\left(y_{p o l y n o m i a l}(t)\right), \text { for } t_{0} \leq t \leq t_{f}
$$

Approach 2. Optimal approach to scanning. The proposed optimal approach developed in Section 3 was applied to the STM scanner. The optimal input for optimal scanning is referred as $u_{f f}^{*}(t)$, composed by the optimal tracking input $u_{t \text { rack }}^{*}(t)$ during $t_{0}<t<t_{i}$ and the optimal transition input is referred to as $u_{t r a n}^{*}(t)$ during $t_{i} \leq t \leq t_{f}$.

\section{Results and Discussions}

The simulation and experimental results provided are for tracking time $\left(T_{\text {track }}\right)$ and transition time $\left(T_{\text {tran }}\right)$ equal to 5 milliseconds $(m s)$ each one $\left(K=\left(T_{\text {tran }} / T_{\text {track }}\right)=1\right)$.

Three approaches were used to find the scan trajectory and the input to the STM: (1) polynomial retrace trajectory with $D C$-gain-based input; (2) polynomial retrace trajectory with inversion-based input; and (3) Optimal scanning approach. Simulation results (inputs and piezo displacements) are shown in Figure (2). For each of the three different approaches, the STM input $\hat{u}$ was found through simulation and applied as feedforward to the STM system. The STM system was used to acquire surface images of a highly oriented pyrolitic graphite (HOPG) sample. Images are shown in Figure (3). All experimental images were obtained with the same settings for the STM, and were obtained one after the other (at one sitting).

Comparison of scanning costs The scanning cost $J_{\text {scanning }}$ with the use of the polynomial retrace trajectory (and inversion-based approach) is compared with the optimal scanning cost in Table (2). 
Table 2: Comparison of scanning cost between polynomial approach to scan trajectory design and optimal scan trajectory design.

\begin{tabular}{|l|c|}
\hline Approach & $K=T_{\text {tran }} / T_{\text {track }}=1$ \\
\hline \hline Polynomial-based & 0.04487 \\
Trajectory design & \\
\hline Optimal scan & 0.02266 \\
Trajectory design & \\
\hline
\end{tabular}
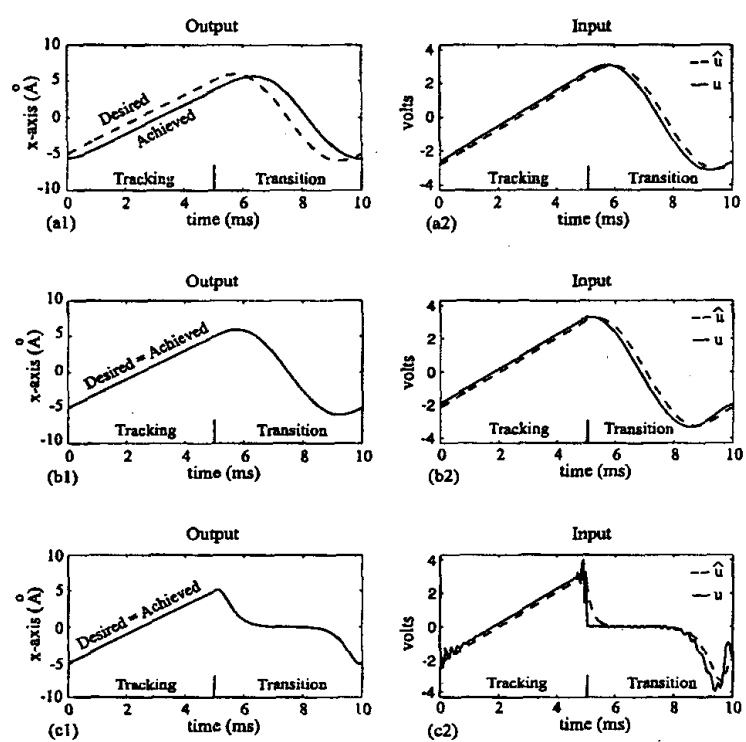

Figure 2: $T_{\text {tran }}=T_{\text {track }}=5 \mathrm{~ms}$, i.e, $K=$ $T_{\text {tran }} / T_{\text {track }}=1$. The left plots are the desired and achieved trajectories. Desired trajectory during the tracking section is a straight line from $y=-5$ to $y=5$ $\dot{A}$. $u$ : input before the pre-filter. $\hat{u}$ : input after the pre-filter. First row: results for the polynomial trajectory with the $D C$-gain approach. Second row: results for the polynomial trajectory with the inversion-based approach. Third row: results for the optimal scan trajectory approach.

\subsection{Discussion of Results}

STM dynamics should be accounted for during scanning. STM dynamics induce vibrations during highspeed scanning. For example, the DC-gain approach does not account for the STM dynamics when finding the input applied during scanning. Therefore, the achieved STM-tip displacement differs from the desired STM-tip displacement as seen in the simulation results Figure (2.a1), which corresponds to a scan rate of $100 \mathrm{~Hz}$. The positioning error with the DC gain approach leads to distortions in the experimentally acquired images (See image for the DC-gain approach in Figure (3)). In contrast, the inversion-based
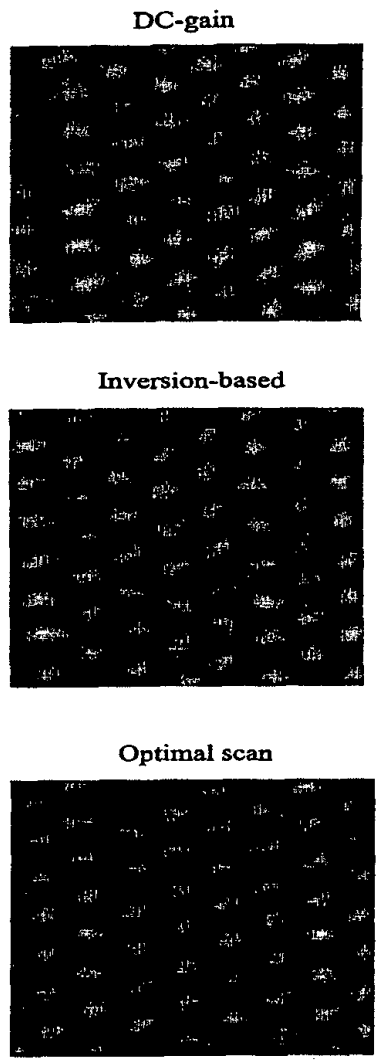

Figure 3: Experimental Results, Images of Graphite Surface: $T_{\text {tran }}=T_{\text {track }}=5 \mathrm{~ms}$.

approach achieves perfect tracking of the same desired output trajectory (compare plots al and b1 in Figure (2)). This is because the inversion-based approach compensates for the dynamics-induced vibrations in the output position. Note that there is no significant increase in the magnitude of the input for achieving this improvement in tracking (compare plots a2 and b2 in Figure (2) and in Figure (2)). Similarly, the model-based optimal scanning approach also achieves perfect tracking of the desired output and therefore the simulations show no tracking error in the output (See Figure (2.c1)). The use of STM-dynamics model leads to removal of the vibration-caused distortions in the images obtained with the inversion-based and optimal-scanning approaches (compare images in Figure (3)).

When the STM is used as a surface analysis instrument, some of these vibration-caused distortions in the image can be removed through post-corrections [20]. However, such distortions limit the use of STMs in real-time visualization of surface processes, and limit the use of STMs in real-time surface modification applications such as nanofabrication [21]. Therefore, compensating for the dynamics is critical to precision positioning during operation of the STM. 
Comparison of Polynomial-based and OptimalScanning Approaches The cost for optimal scanning input should always be lower than the cost for inversion-based approach because the polynomial-based retrace is one of the possible trajectories over which the optimization of the input-energy is performed - this is reflected in Table 2 which compares the input cost with the two approaches. The optimal-scanning approach requires less than balf the input energy required by the polynomial-based approach. Note that the optimal-scanning approach tends to keep the input zero during a significant portion of the retrace section (approximately $3 \mathrm{~ms}$ ) to minimize the input energy (compare plots b2 and c2 in Figure (2)). This implies that the transition time could be reduced without significant increase in the input energy - reduction of the transition time from $5 \mathrm{~ms}$ to $2 \mathrm{~ms}$. Note that both the approaches lead to exact output tracking during the active scanning as seen in the experimentally obtained images (rows 2 and 3 in Figure (3)); however, the optimal-scanning approach requires less input-energy and it provides a systematic approach to the design and control of the output trajectory for scanning.

\section{Conclusions}

The problem of designing the output trajectory for a scanner such that the input energy is minimized was posed and solved in this article. The problem was solved by integrating (a) standard optimal state-transition approach during the output-transition (retrace) section with (b) a model-based inversion approach to maintain perfect output-tracking during the active-scan (tracking) section. The cost for the entire scan (active scan and retrace) was then minimized to solve the optimal scanning problem. The approach was applied to a Scanning Tunneling Microscope, and simulation and experimental results were presented to illustrate the technique and its advantages.

\section{References}

[1] D. Croft and S. Devasia. Vibration compensation for high speed scanning tunneling microscopy. Review of Scientific Instruments, 70(12):4600-4605, December 1999.

[2] R.-F. Fung, J. S. Huang, C. G. Chien, and Y. C. Wang. Design and application of a continuous repetitive controller for rotating mechanisms. International Journal of Mechanical Sciences, 42:1805-1819, 2000.

[3] C. Cloet, M. Tomisuka, and R. Horowitz. Design requirements and reference trajectory generation for a copier paperpath. IEEE/ASME International Conference on $A d-$ vanced Intelligent Mechatronics Proceedings, pages 911-916, July 2001. Como, Italy.

[4] M. A. Arteaga and B. Siciliano. On tracking control of flexible robot arms. IEEE Trans. Autom. Control, 45(3):520-527, 2000.

[5] A. Piazzi and A. Visioli. Minimum-time systeminversion-based motion planning for residual vibration reduction. IEEE/ASME Trans. on Mechatronics, 5(1):12-22; March 2000.

[6] A. V. Dowd and M. D. Thanos. Vector motion processing using spectral windows. IEEE Control Systems Magazine, 20(5):8-19, October 2000.

[7] G. M. Whitesides and H. C. Love. The art of building small. Scientific American, September, 285(3):39-47, 2001.

[8] R. Wiesendanger, editor. Scanning Probe Microscopy and Spectroscopy. Cambridge University Press, 1994.

[9] A. Alleyne and M. Pomykalski. Control of a class of nonlinear systems subject to periodic exogenous signals. IEEE Trans. Control Systems Technology, 8(2):279-287, March 2000.

[10] J. Ghosh and B Paden. Nonlinear repetitive control. IEEE Trans. Autom. Control, 45(5):949-954, May 2000.

[11] J. H. Lee, S. Natarajan, and K. S. Lee. A modelbased predictive control approach to repetitive control of continuous processes with periodic operations. Journal of Process Control, 11:195-207, 2001.

[12] F. L. Lewis and V. L. Syrmos. Optimal Control John Wiley \& Sons, Inc., Third Avenue, New York, NY 101580012, 1995.

[13] S. Sastry. Nonlinear Systems. Analysis, Stability and Control, volume 10 of Interdisciplinary applied mathematics. Springer-Verlag New York, Inc, 175 Fifth Avenue, New York, NY 10010, USA, 1999.

[14] Q. Zou and S. Devasia. Preview-based stableinversion for output tracking of linear systerns. ASME J. Dyn. Syst., Meas., Control, pages 625-630, December 1999. [15] L. M. Silverman. Inversion of multivariable linear systems. IEEE Trans. Autom. Control, 14(3):270-276, 1969.

[16] M. K. Sain and J. L. Massey. Invertibility of linear time-invariant dynamical systems. IEEE Trans. Autom. Control, 14:141-149, 1969.

[17] S. Devasia, D. Chen, and B. Paden. Nonlinear inversion-based output tracking. IEEE Trans. Autom. Control, 41(7):930-942, 1996.

[18] J. M. Ortega. Matrix Theory. The university series in mathematics. 1987 Plenum Press, New York., 233 Spring Street, New York, N. Y. 10013, 1987.

[19] N. K. Gupta. Frequency-shaped cost functionals: Extension of linear-quadratic-gaussian design methods. Journal of Guidance and Control, 3(6):529-535, Nov 1980.

[20] R. Barrett and C. Quate. Optical scan-correction system applied to atomic force microscopy. Review of Scientific Instruments, 62(6):1393-1399, 1991.

[21] E. Snow and F. Perkins. Nanofabrication with proximal probes. Proceedings of the IEEE, 85:601-611, April 1997. 